\title{
Urine Lunx mRNA as a Potential Molecular Diagnostic Tool for Lung Cancer
}

\author{
Sanxia Zhao1 ${ }^{1}$, Yinlin $\mathrm{Ge}^{1}$
}

\begin{abstract}
${ }^{1}$ Department of Biochemistry and Molecular Biology, Medical College, Qingdao University, Qingdao, 266000, Shandong, China

Abstract: Objective: Exploring the expression of cell-free RNA in urine of normal patients and lung cancer patients, analyze its clinical value in the dignosis of lung cancer. Methods: The urine samples were collected from lung cancer patients and healthy people. Extracted the total cf-RNA from all samples. The contents of total cf-RNA in two groups were detected, and the expressions of LUNX mRNA in two groups were measured by quantitative real-time PCR. Receiver-operating characteristics (ROC) curve was established to evaluate the diagnostic value of urine cf-RNA for the differentiation between lung cancer patients and the control group. Results: Compare to the normal group $(50.50 \pm 4.527 \mathrm{ng})$, the urinary cf-RNA content in patients $(133.8 \pm 8.615 \mathrm{ng})$ was significantly increased, $\mathrm{p}<0.05$. The expression of urinary lunx mRNA level from patients was raised, $p<0.05$. ROC curve analysis showed that, in the diagnosis, AUC value of lunx mRNA was 0.83(95\% CI, 0.747-0.906), p<0.05. Conclusion: The expression of urine cf-RNA and Lunx mRNA was elevated, and can be used as a potential molecular diagnostic tool for lung cancer.
\end{abstract}

Keywords: Urine, Lung Cancer, cf-RNA, Diagnosis

\section{Introduction}

Lung cancer is a prevalent malignant tumor of high incidence and mortality. It is one of the most dangerous diseases to human health and life. Due to the lack of convenient and efficient cancer screening methods and insufficient cognition of early diagnosis and treatment, the incidence and mortality rate of lung cancer in China are the highest among all cancers ${ }^{1}$. Therefore, it is very important to find convenient and specific biomarkers for early lung cancer ${ }^{2}$. In recent years, with the rise of precision medicine and liquid biopsy, the advantages of non-invasive detection in disease prevention and prognosis monitoring have been gradually recognized 3. Urine detection is a truly non-invasive detection method. A research by Zhao shows that free RNA in urine is relatively stable, which means that free RNA in urine can be used as a potential biomarker for early discovering diseases ${ }^{4}$.

\section{Experimental material and method 2.1 Clinical urine samples}

Urine samples from 58 lung cancer patients were collected in this study. All the patients were diagnosed in the Affiliated Hospital of Qingdao University. There are 36 males and 22 females, aged $35-76$ with a median age of 56 . And 35 cases in the control group, including 21 males and 14 females, aged 32-65 with a median age of 52 .

\subsection{Total urinary RNA extraction}

All of the urine samples from patients and control groups were centrifuged at $5000 \mathrm{r} / \mathrm{min}$ for $40 \mathrm{~min}$ at $4^{\circ} \mathrm{C}$. Then collected the supernatant fluid and filtered it through a positively charged nylon membrane to absorb the nucleic acid onto the nylon membrane. Then the membrances were drying in a $56^{\circ} \mathrm{C}$ drying oven for 5 minutes. The nylon membrane was cut into small pieces and collected into $1.5 \mathrm{ml}$ Ep tube without RNA enzyme ${ }^{5}$. Put the tubes at $-80^{\circ} \mathrm{C}$. Total RNA in urine was adsorbed onto the membrances now. Then extracted them by using TRIzol reagent (Takara, China). The content of RNA was detected by a nanodrop, and the difference of total RNA between patients and controls should be analyzed.

\subsection{Reverse transcription and real-time qPCR}

The total RNA was transcribed into cDNA after the content detection (TransScript One-step gDNA Removal and cNA Synthesis SuperMix, China). Then amplified by fluorescent quantitative PCR (TransStart Tip Green qPCR SuperMix, China). The conditions were as follows: $94^{\circ} \mathrm{C}$ for $30 \mathrm{~s}, 94^{\circ} \mathrm{C}$ for $5 \mathrm{~S}, 60^{\circ} \mathrm{C}$ for 30s, 40 cycles. All samples were calculated as exponential of $2^{-\Delta \Delta C t}$. The primer sequence are listed in table 1 . 
Table 1. Primer sequence

\begin{tabular}{ccc}
\hline gene symbol & forward primer & reverse primer \\
\hline GAPDH & 5'-AGAAGGCTGGGGCTCATTTG-3' & 5'-AGGGGCCATCCACAGTCTTC-3' \\
Lunx & 5'-AAGTCTGTTGAGGCTGGCTG-3' & 5'-GCCAAGTCCAT CAAGCAGAG-3' \\
\hline
\end{tabular}

\subsection{ROC curve analysis}

ROC curve was established to evaluate the diagnostic value of urine cf-RNA for the differentiation between lung cancer patients and control group.

\subsection{Statistical analysis}

The Statistical analyses were performed with Prism 6.0 and MedCal 17 statistical software. All values were depicted as mean $( \pm)$ standard deviation (SD) and are considered significant if $\mathrm{p}<0.05$.

\section{Results}

\subsection{Urinary total RNA}

The average amount of urinary total RNA from patients was $133.8 \pm 8.615 \mathrm{ng}$ whereas it was $50.50 \pm 4.527 \mathrm{ng}$ from the normal group. The total RNA amount of patients was increased $(\mathrm{P}<0.05$, Fig. 1).

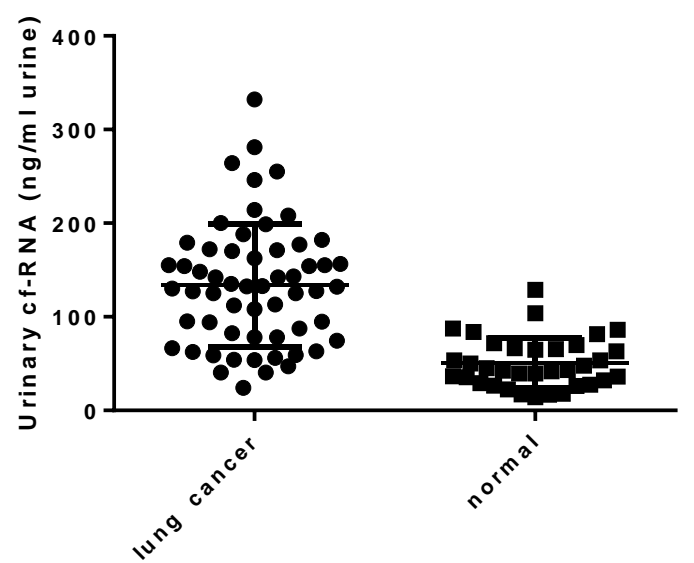

Figure 1. Urinary total RNA from lung cancer patients and normal people

\section{2 qPCR results}

Urinary lunx mRNA was amplified by qPCR. The expression level of urinary lunx mRNA from patients was increased, $\mathrm{p}<0.05$.

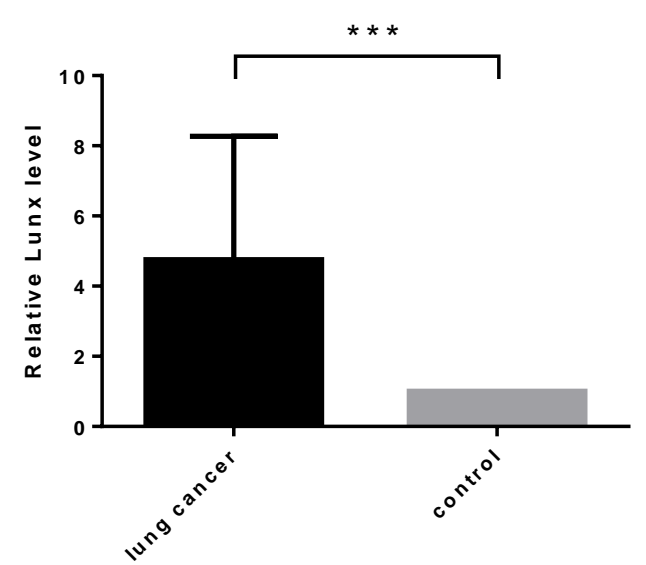

Figure 2. Expression levels of Lunx mRNA in urine samples of lung cancer and control group.

We measured the expression levels of Lunx mRNA from 58 patients and 35 normal people by qPCR, RNA levels were normalized to GAPDH。***: $\mathrm{P}<$ 0.001

3.3 The diagnostic value of urinary Lunx mRNA To assess whether urinary Lunx mRNA expression can be used as a diagnostic tool for lung cancer, we use ROC curves for analysis (Fig. 4). The area under Lunx mRNA curve (AUC) was 0.838 (95\% CI: $0.747-0.906$ ), the Jordan index was 0.628 , with a sensitivity of 91.38 and specificity of 71.43 .

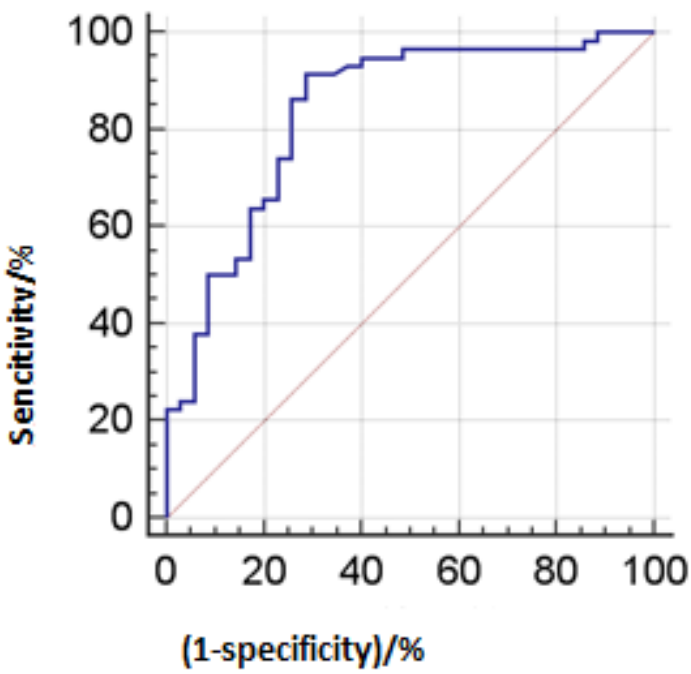

Figure 3. Receiver operating characteristic (ROC) curve analysis by Lunx mRNA 


\section{Discussion}

Lunx mRNA, which encodes human lung-specific protein $x$ (LUNX), is located at 20p11.1-q12. The gene is specifically expressed in lung tissue while other tissues did not. Recent years, there were reports of lunx mRNA expression in the blood too ${ }^{6}$.

In this study, we analyzed the expression of the cf-RNA in urine, and found that the urinary total cf-RNA content and the urinary lunx mRNA expression level in the patients were both significantly increased. The ROC curve analysis showed that lunx mRNA had good sensitivity and sensitivity in the diagnosis of lung cancer and could be used as a potential diagnostic biomarker.

Lung cancer usually takes a long time from the onset of disease, clinical symptoms to diagnosis. Therefore, if the disease can be detected as early as possible and then treated, it can effectively improve the survival rate of lung cancer. At present, the study of liquid life is more focused on the free nucleic acid in the blood $7 ; 8$. Because of the inherent stability regulation mechanism of the body, the early changes in the blood are easy to be removed, which is not conducive to detection ${ }^{9}$. There is no such mechanism in urine. Early changes are more sensitive and easy to capture 10 . Urine detection as a routine physical examination items, sampling is convenient and truly non-invasive testing, if the urine can be found in high-efficiency, sensitive biomarkers, it will be able to effectively detect early disease, thus early treatment, improve the survival rate. However, the content of free RNA in urine is low, and further research is needed to extract high quality RNA efficiently and concisely, as well as to screen molecular markers with high sensitivity and specificity.

\section{References}

1. Xu-Welliver M, Carbone D P. Blood-based biomarkers in lung cancer: prognosis and treatment decisions[J]. Translational lung cancer research, 2017, 6(6): 708.

2. Chen Y Q, Li D M, Cai Y Y, et al. The expression of survivin messenger RNA in sputum and cancerous tissue in human lung cancer $[\mathrm{J}]$. CHINESE JOURNAL OF TUBERCULOSIS AND RESPIRATORY DISEASES, 2005, 28(4):225-229.

3. Jing J, Gao Y H. Urine as a Source of Novel Biomarkers [J]. Progress in Biochemistry and Biophysics, 2016, 43(11): 1019-1028.

4. Zhao A, Péoc'h M, Cottier M, et al. Cell-free RNA content in urine as a possible molecular diagnostic tool for clear cell renal cell carcinoma[J]. International journal of cancer, 2015, 136(11): 2610-2615.

5. Zhang F, Cheng X, Yuan Y, et al. Urinary microRNA can be concentrated, dried on membranes and stored at room temperature in vacuum bags[J]. PeerJ, 2015, 3: e1082.

6. Zhao $\mathrm{W} J$, Zhou $\mathrm{H} \mathrm{X}$, Wang $\mathrm{S} \mathrm{J}$ et al. The clinical significance of Lunx mRNA detection in plasma and peripheral mononuclear cells in patients with lung cancer [J]. LABORATORY MEDICINE, 2012, 27(8):631-634.
7. Kapellos G, Polonifi K, Farmakis D, et al. Overexpression of survivin levels in circulation and tissue samples of lung cancer patients[J]. Anticancer research, 2013, 33(8): 3475-3480.

8. Zhao W, Zhao J J, Zhang L, et al. Serum miR-21 level: a potential diagnostic and prognostic biomarker for non-small cell lung cancer[J]. International journal of clinical and experimental medicine, 2015, 8(9): 14759.

9. Kao H W, Pan C Y, Lai C H, et al. Urine miR-21-5p as a potential non-invasive biomarker for gastric cancer[J]. Oncotarget, 2017, 8(34): 56389.

10. Wang C,Li Y,Fang X D. Research progress on liquid biopsy in oncology and its clinical applications. Hereditas(Beijing), 2017, 39(3): 220-231 